ESAIM: COCV 22 (2016) 539-542

DOI: $10.1051 / \mathrm{cocv} / 2016005$
ESAIM: Control, Optimisation and Calculus of Variations

www.esaim-cocv.org

\title{
ERRATUM TO THE ARTICLE HAMILTON-JACOBI EQUATIONS FOR OPTIMAL CONTROL ON JUNCTIONS AND NETWORKS
}

\author{
Yves Achdou ${ }^{1}$, Salomé Oudet ${ }^{2}$ And Nicoletta TChou ${ }^{2}$
}

\begin{abstract}
We correct a mistake which affects an intermediate result, namely the second part of Lemma 4.5. The main results of the article are unchanged.
\end{abstract}

Mathematics Subject Classification. 34H05, 49J15.

Received November 3, 2015. Revised January 2, 2016.

Published online March 18, 2016.

ESAIM: COCV 21 (2015) 876-899. Doi:10.1051/cocv/2014054

The second part of Lemma 4.5, concerning subsolutions, is not correct in the published version of the paper. Recall that we are interested in proving a comparison principle for sub and super solutions of

$$
\lambda u(x)+\sup _{(\zeta, \xi) \in \mathrm{FL}(x)}\{-D u(x, \zeta)-\xi\}=0 \quad \text { in } \mathcal{G} .
$$

Lemma 4.5 must be modified as follows:

Lemma 4.5. Let $v: \mathcal{G} \rightarrow \mathbb{R}$ be a viscosity supersolution of (3.1) in $\mathcal{G}$. Then if $x \in J_{i} \backslash\{0\}$, we have for all $t>0$

$$
v(x) \geq \inf _{\alpha_{i}(\cdot), \theta_{i}}\left(\int_{0}^{t \wedge \theta_{i}} \ell_{i}\left(y_{x}^{i}(s), \alpha_{i}(s)\right) \mathrm{e}^{-\lambda s} \mathrm{~d} s+v\left(y_{x}^{i}\left(t \wedge \theta_{i}\right)\right) \mathrm{e}^{-\lambda\left(t \wedge \theta_{i}\right)}\right),
$$

where $\alpha_{i} \in L^{\infty}\left(0, \infty ; A_{i}\right)$, yx $y_{x}^{i}$ is the solution of $y_{x}^{i}(t)=x+\left(\int_{0}^{t} f_{i}\left(y_{x}^{i}(s), \alpha_{i}(s)\right) \mathrm{d} s\right) e_{i}$ and $\theta_{i}$ is such that $y_{x}^{i}\left(\theta_{i}\right)=0$ and $\theta_{i}$ lies in $\left[\tau_{i}, \bar{\tau}_{i}\right]$, where $\tau_{i}$ is the exit time of $y_{x}^{i}$ from $J_{i} \backslash\{O\}$ and $\bar{\tau}_{i}$ is the exit time of $y_{x}^{i}$ from $J_{i}$.

Remark. Concerning subsolutions, the comparison results of Barles-Perthame [2] imply the following suboptimality principle for subsolutions that will not be needed in the sequel: let $w$ be a continuous viscosity

Keywords and phrases. Optimal control, networks, Hamilton-Jacobi equations, viscosity solutions.

1 Université Paris Diderot, Sorbonne Paris Cité, Laboratoire Jacques-Louis Lions, UMR 7598, UPMC, CNRS, 75205 Paris,

France. achdou@ljll.univ-paris-diderot.fr

2 IRMAR, Université de Rennes 1, Rennes, France. nicoletta.tchou@univ-rennes1.fr 
subsolution of (3.1) in $\mathcal{G}$. If $x \in J_{i} \backslash\{0\}$, we have for all $t>0$,

$$
w(x) \leq \inf _{\alpha_{i}(\cdot)} \sup _{\theta_{i}}\left(\int_{0}^{t \wedge \theta_{i}} \ell_{i}\left(y_{x}^{i}(s), \alpha_{i}(s)\right) \mathrm{e}^{-\lambda s} \mathrm{~d} s+w\left(y_{x}^{i}\left(t \wedge \theta_{i}\right)\right) \mathrm{e}^{-\lambda\left(t \wedge \theta_{i}\right)}\right),
$$

where $\alpha_{i} \in L^{\infty}\left(0, \infty ; A_{i}\right), y_{x}^{i}$ is the solution of $y_{x}^{i}(t)=x+\left(\int_{0}^{t} f_{i}\left(y_{x}^{i}(s), \alpha_{i}(s)\right) \mathrm{d} s\right) e_{i}$ and $\theta_{i}$ is such that $y_{x}^{i}\left(\theta_{i}\right)=0$ and $\theta_{i}$ lies in $\left[\tau_{i}, \bar{\tau}_{i}\right]$, where $\tau_{i}$ is the exit time of $y_{x}^{i}$ from $J_{i} \backslash\{O\}$ and $\bar{\tau}_{i}$ is the exit time of $y_{x}^{i}$ from $J_{i}$.

Then, Theorem 4.6 should be very slightly modified as follows (the very minor changes in the proof do not need to be written):

Theorem 4.6. Assume [H0], [H1], [H2] and [H3]. Let $r>0$ be given by Lemma 4.2: any bounded subsolution of (3.1) is Lipschitz continuous in $B(O, r) \cap \mathcal{G}$. Let $v: \mathcal{G} \rightarrow \mathbb{R}$ be a viscosity supersolution of (3.1), bounded from below by $-c|x|-C$ for two positive numbers $c$ and $C$. Either $[\mathrm{A}]$ or $[\mathrm{B}]$ below is true:

[A] There exists a sequence $\left(\eta_{k}\right)_{k \in \mathbb{N}}$ of positive real numbers such that $\lim _{k \rightarrow+\infty} \eta_{k}=\eta>0$, an index $i \in$ $\{1, \ldots, N\}$ and a sequence $x_{k} \in J_{i}$ such that $x_{k} \in J_{i} \backslash\{O\}$ and $\lim _{k \rightarrow+\infty} x_{k}=O$ satisfying the following: for any $k \in \mathbb{N}$, there exists a control law $\alpha_{i}^{k}$ such that the corresponding trajectory $y_{x_{k}}$ remains in $J_{i} \cap B(O, r)$ in the time interval $\left[0, \eta_{k}\right]$, i.e. $y_{x_{k}}(s) \in J_{i} \cap B(O, r)$ for all $s \in\left[0, \eta_{k}\right]$, and is such that

$$
v\left(x_{k}\right) \geq \int_{0}^{\eta_{k}} \ell_{i}\left(y_{x_{k}}(s), \alpha_{i}^{k}(s)\right) \mathrm{e}^{-\lambda s} \mathrm{~d} s+v\left(y_{x_{k}}(\eta)\right) \mathrm{e}^{-\lambda \eta_{k}}
$$

$[\mathrm{B}]$

$$
\lambda v(O)+H_{O}^{T} \geq 0
$$

A new lemma is needed to replace the second part of Lemma 4.5:

Lemma 4.7. Assume [H0], [H1], [H2] and [H3]. Let $r>0$ be given by Lemma 4.2: any bounded subsolution of (3.1) is Lipschitz continuous in $B(O, r) \cap \mathcal{G}$. Consider $i \in\{1, \ldots, N\}, x \in\left(J_{i} \backslash\{O\}\right) \cap B(O, r), \alpha_{i} \in$ $L^{\infty}\left(0, \infty ; A_{i}\right)$. Let $\eta>0$ be such that $y_{x}(t)=x+\left(\int_{0}^{t} f_{i}\left(y_{x}(s), \alpha_{i}(s)\right) \mathrm{d} s\right) e_{i}$ belongs to $J_{i} \cap B(O, r)$ for any $t \in[0, \eta]$. For any bounded viscosity subsolution $v$ of $(3.1)$,

$$
v(x) \leq \int_{0}^{\eta} \ell_{i}\left(y_{x}(t), \alpha_{i}(t)\right) \mathrm{e}^{-\lambda t} \mathrm{~d} t+v\left(y_{x}(\eta)\right) \mathrm{e}^{-\lambda \eta} .
$$

Proof. Since $v$ is Lipschitz continuous in $B(O, r) \cap J_{i}$, the function $t \mapsto v\left(y_{x}(t)\right) \mathrm{e}^{-\lambda t}$ is Lipschitz continuous in $[0, \eta]$. Let us define the sets $K_{O}=\left\{t \in(0, \eta): y_{x}(t)=O\right\}$ and $K_{O}^{c}=[0, \eta] \backslash K_{O}$. It is clear that $K_{O}$ is closed and that $K_{O}^{c}$ is an open subset of $[0, \eta]$. We first observe that, from Stampacchia's theorem,

$$
\left.\int_{0}^{\eta} 1_{K_{O}}(t) \frac{\mathrm{d}}{\mathrm{d} t}\left(v\left(y_{x}(t)\right) \mathrm{e}^{-\lambda t}\right)\right) \mathrm{d} t=-\lambda v(O) \int_{0}^{\eta} 1_{K_{O}}(t) \mathrm{e}^{-\lambda t} \mathrm{~d} t
$$

Therefore, we deduce from Lemma 4.3 that

$$
\left.\int_{0}^{\eta} 1_{K_{O}}(t) \frac{\mathrm{d}}{\mathrm{d} t}\left(v\left(y_{x}(t)\right) \mathrm{e}^{-\lambda t}\right)\right) \mathrm{d} t \geq H_{O}^{T} \int_{0}^{\eta} 1_{K_{O}}(t) \mathrm{d} t \geq-\int_{0}^{\eta} \ell_{i}\left(O, \alpha_{i}(t)\right) 1_{K_{O}}(t) \mathrm{d} t=-\int_{0}^{\eta} \ell_{i}\left(y_{x}(t), \alpha_{i}(t)\right) 1_{K_{O}}(t) \mathrm{d} t .
$$

On the other hand, since $K_{O}^{c}$ is an open subset of $[0, \eta]$, there exists a countable family of disjoint intervals $\left(\omega_{j}\right)_{j \in J}, \omega_{j} \subset[0, \eta]$ such that $K_{O}^{c}=\bigcup_{j \in J} \omega_{j}$. Let $a_{j}<b_{j}$ be the lower and upper endpoints of $\bar{\omega}_{j}$. We can assume that $\left[a_{j}, b_{j}\right] \cap\left[a_{k}, b_{k}\right]=\emptyset$ if $j \neq k$. 
From a classical suboptimality principle, see ([1], Thm. III.2.33), we see that for any $j \in J$,

$$
v\left(y_{x}\left(b_{j}\right)\right) \mathrm{e}^{-\lambda b_{j}}-v\left(y_{x}\left(a_{j}\right)\right) \mathrm{e}^{-\lambda a_{j}} \geq-\int_{a_{j}}^{b_{j}} \ell_{i}\left(y_{x}(t), \alpha_{i}(t)\right) \mathrm{e}^{-\lambda t} \mathrm{~d} t .
$$

Noting that

$$
v\left(y_{x}\left(b_{j}\right)\right) \mathrm{e}^{-\lambda b_{j}}-v\left(y_{x}\left(a_{j}\right)\right) \mathrm{e}^{-\lambda a_{j}}=\int_{0}^{\eta} \frac{\mathrm{d}}{\mathrm{d} t}\left(v\left(y_{x}(t)\right) \mathrm{e}^{-\lambda t}\right) 1_{\left(a_{j}, b_{j}\right)}(t) \mathrm{d} t,
$$

and summing over $j \in J$, we obtain that

$$
\left.\int_{0}^{\eta} 1_{K_{O}^{c}}(t) \frac{\mathrm{d}}{\mathrm{d} t}\left(v\left(y_{x}(t)\right) \mathrm{e}^{-\lambda t}\right)\right) \mathrm{d} t \geq-\int_{0}^{\eta} \ell_{i}\left(y_{x}(t), \alpha_{i}(t)\right) 1_{K_{O}^{c}}(t) \mathrm{d} t .
$$

We get (a) by summing (b) and (c).

The main comparison result holds but its proof is modified.

Theorem 5.1. Assume [H0], [H1], [H2] and [H3]. Let $u: \mathcal{G} \rightarrow \mathbb{R}$ be a bounded viscosity subsolution of (3.1), and $v: \mathcal{G} \rightarrow \mathbb{R}$ be a bounded viscosity supersolution of (3.1). Then $u \leq v$ in $\mathcal{G}$.

Proof. It is a simple matter to check that there exists a positive real number $M$ such that the function $\psi(x)=$ $-|x|^{2}-M$ is a viscosity subsolution of (3.1.). For $0<\mu<1, \mu$ close to 1 , the function $u_{\mu}=\mu u+(1-\mu) \psi$ is a viscosity subsolution of (3.1), which tends to $-\infty$ as $|x|$ tends to $+\infty$. Let $M_{\mu}$ be the maximal value of $u_{\mu}-v$ which is reached at some point $\bar{x}_{\mu}$.

We want to prove that $M_{\mu} \leq 0$.

(1) If $\bar{x}_{\mu} \neq O$, then we introduce the function $u_{\mu}(x)-v(x)-d^{2}\left(x, \bar{x}_{\mu}\right)$, which has a strict maximum at $\bar{x}_{\mu}$, and we double the variables, i.e. for $0<\varepsilon \ll 1$, we consider

$$
u_{\mu}(x)-v(y)-d^{2}\left(x, \bar{x}_{\mu}\right)-\frac{d^{2}(x, y)}{\varepsilon^{2}} .
$$

Classical arguments then lead to the conclusion that $u_{\mu}\left(\bar{x}_{\mu}\right)-v\left(\bar{x}_{\mu}\right) \leq 0$, thus $M_{\mu} \leq 0$.

(2) If $\bar{x}_{\mu}=O$. We use Theorem 4.6; we have two possible cases:

[B] $\lambda v(O) \geq-H_{O}^{T}$.

From Lemma 4.3, $\lambda u(O)+H_{O}^{T} \leq 0$. Therefore, we obtain that $u_{\mu}(O) \leq v(O)$, thus $M_{\mu} \leq 0$.

[A] With the notations of Theorem 4.6,

$$
v\left(x_{k}\right) \geq \int_{0}^{\eta_{k}} \ell_{i}\left(y_{x_{k}}(s), \alpha_{i}^{k}(s)\right) \mathrm{e}^{-\lambda s} \mathrm{~d} s+v\left(y_{x_{k}}\left(\eta_{k}\right)\right) \mathrm{e}^{-\lambda \eta_{k}} .
$$

Moreover, since $y_{x_{k}}(s) \in J_{i} \cap B(O, r)$ for all $s \in\left[0, \eta_{k}\right]$, Lemma 4.7 can be applied and yields that

$$
u_{\mu}\left(x_{k}\right) \leq \int_{0}^{\eta_{k}} \ell_{i}\left(y_{x_{k}}(s), \alpha_{i}^{k}(s)\right) \mathrm{e}^{-\lambda s} \mathrm{~d} s+u_{\mu}\left(y_{x_{k}}\left(\eta_{k}\right)\right) \mathrm{e}^{-\lambda \eta_{k}}
$$

Therefore

$$
u_{\mu}\left(x_{k}\right)-v\left(x_{k}\right) \leq\left(u_{\mu}\left(y_{x_{k}}\left(\eta_{k}\right)\right)-v\left(y_{x_{k}}\left(\eta_{k}\right)\right)\right) \mathrm{e}^{-\lambda \eta_{k}} .
$$

Letting $k$ tend to $+\infty$, we find that $M_{\mu} \leq M_{\mu} \mathrm{e}^{-\lambda \eta}$, which implies that $M_{\mu} \leq 0$

We conclude by letting $\mu$ tend to 1 . 


\section{REFERENCES}

[1] M. Bardi and I. Capuzzo-Dolcetta, Optimal Control and Viscosity Solutions of Hamilton-Jacobi-Bellman Equations. Systems and Control: Foundations and Applications. With appendices by Maurizio Falcone and Pierpaolo Soravia. Birkhäuser Boston Inc., Boston, MA (1997).

[2] G. Barles and B. Perthame, Comparison principle for Dirichlet-type Hamilton-Jacobi equations and singular perturbations of degenerated elliptic equations. Appl. Math. Optim. 21 (1990) 21-44. 